\title{
Statement of Argentine pediatric endocrinologists on growth hormone interchangeability
}

\section{INTRODUCTION}

Growth hormone $(\mathrm{GH})$, just like vaccines, antibodies, and other hormones, is a biological medicinal product (BMP); therefore, it is produced by a complex mechanism that is specific to each (trademark) product. BMPs are subjected to prolonged, comprehensive assessment processes and must be approved prior to their use. The patents of early recombinant human GHs are starting to expire, and this has encouraged pharmaceutical companies to manufacture GH in accordance with the processes corresponding to the original products, thus resulting in biosimilar recombinant GH. In Argentina, there are seven different GH trademarks; only one is a biosimilar product: Omnitrope ${ }^{\circledR}$ (Sandoz) is biosimilar to Genotropin ${ }^{\circledR}$ (Pfizer). Other products available in the market include $\mathrm{HHT}^{\circledR}$ (Biosidus), Hutrope ${ }^{\circledR}$ (Elly Lilly), NorditropinFlexpro ${ }^{\circledR}$ (Novo Nordisk), Saizen ${ }^{\circledR}$ (Merck), and Zomacton ${ }^{\circledR}$ (Ferring).

For an adequate understanding of this article, it is important to outline the characteristics of the different BMPs. ${ }^{1-5}$

BMP: the drug substance of this medicine is a biological substance (e.g., recombinant deoxyribonucleic acid [DNA], vaccines, plasmaor blood-related products, and monoclonal antibodies). A biological substance is produced or obtained from a biological source and, for its characterization, requires a combination of physicochemical and/or biological tests, as well as a completely developed and controlled manufacturing process.

Reference biological medicinal product (RBMP): a BMP that has been approved and marketed in the European Union (EU), ${ }^{6}$ the USA or other countries.

Similar biological medicinal product (SBMP): a biological drug produced by a new manufacturer using a process that is similar to that of a known RBMP. The quality, safety, and effectiveness profiles of a SBMP are comparable to those of a RBMP, and it has been approved by the regulatory agencies of each country by means of an abbreviated process.

Health care providers are responsible for patient care and, therefore, for the treatments they prescribe, so it is critical that they have reliable information on biological drugs, their clinical development, approval, and safety monitoring.
The objective of this article is not to provide a detailed description of all the characteristics of a (reference or similar) biological drug, but to mention the basic concepts and then discuss the potential for $\mathrm{GH}$ interchangeability and point out which is the correct stance of pediatric endocrinologists on this aspect.

Annex I describes the basic concepts about biological drugs. (Spanish version)

\section{Interchange ability of biological products and similar products}

Based on the characteristics of biosimilar drugs, quality, safety, and effectiveness profiles are comparable to those of the reference product. Therefore, they would be valid therapeutic alternatives that could be used instead of reference products.

Most biological drugs are targeted for longterm use in chronic conditions. The change from a reference product to the corresponding biosimilar drug would be greatly relevant in terms of both costs and promotion of new technologies tending to favor patients' access to future biological products.

It is important to establish the difference between drug interchangeability and switching. Interchangeability means that the reference biological drug may be replaced with its biosimilar drug without the intervention of the health care provider who prescribed the reference drug. However, switching refers to using an alternative drug prescribed by the health care provider.

The document "Interchangeability among growth hormone biosimilar drugs" ("Intercambiabilidad entre biosimilares de la hormona de crecimiento"), developed in the setting of the agreement "Counseling the Secretariat of Health Promotion, Prevention, and Risk Control. National Ministry of Health for the Assessment of Health Technologies and Evidence-Informed Decision-Making," in August 2017, refers to the recombinant human GHs available in Argentina: "Given that, during the manufacturing process, small variations occur among manufacturers due to the complexity and production methods used, the final product has small differences and the resulting biological products are not identical. It was not possible to find clinical trials on the interchangeability with 
other trademark products available in Argentina".

Several scientific communications have been issued on biosimilar drug interchangeability. Annex II details the main references to studies on interchangeability related to $\mathrm{GH}$.

\section{Health care providers' stance on growth hormone}

Although there are no studies that demonstrate the absolute superiority of one product over another, it is clear that each product has its own particular features. However, the current evidence about their effectiveness suggests that all products have demonstrated non-inferiority. It is also necessary to point out that the available bibliography does not include studies done in a significant number of patients to support, in a solid and conclusive manner, that replacing biological drugs will not cause mildly- or highlysevere untoward side-effects on the patient's health in the long term.

It is worth noting that the present stance of health care providers on biosimilar drug interchangeability is framed within the thorough understanding of the currently valid legislation in Argentina in relation to the Regulation of Drug Prescriptions. ${ }^{78}$

The recommendation to providers responsible for the health of our patients is to reject $\mathrm{GH}$ interchangeability without the consent of the prescribing physician.

This recommendation is based on the following:

a) The scientific evidence on the biosafety of GH interchangeability is scarce, based on immunogenicity and adverse event reporting, and limited to few years of treatment. The European Community has published a 10 -year safety monitoring report. There is little information available on the longterm outcomes of GH therapy, especially in individuals treated during childhood. In the long term, reports include potential increased risks for cancer and a strong relation between hemorrhagic stroke and GH therapy during childhood for isolated GH deficiency or pediatric short stature..$^{9-11}$

b) Therefore, it is of vital importance to have an adequate drug surveillance program that includes the non-interchangeability of GH used in a vulnerable population, like children. It will only be possible to identify biological drugrelated long-standing serious adverse events if most variables are controlled, one of them being the drug used during the treatment. c) In this setting, where long-term biosafety of $\mathrm{GH}$ therapy in the pediatric age still calls for future investigations, it is worth recalling that the pediatric endocrinologist in charge of the patient is legally responsible for his/ her treatment. For what is understood, it is not acceptable for any financer, including the state, to carry out GH interchangeability without the explicit consent of the health care provider in charge of the patient.

d) Daily GH administration is always associated with the use of an application device (approved by the National Drug, Food and Technology Administration of Argentina [Administración Nacional de Medicamentos, Alimentos y Tecnología Médica, ANMAT]), which varies depending on the trademark. Since GH is administered by the patients themselves or their parents at home, all devices require that both patients and their families are thoroughly trained. Therefore, product interchangeability clearly increases the potential for $\mathrm{GH}$ administration errors (dosage, form of administration, etc.), together with the predictable negative consequences for the patient. Currently, the average GH therapy lasts about 5 years (range: approximately 3-15 years). In this regard, it is worth mentioning that the device is provided to patients at no cost but, when changing from one brand to another, this may not happen and it would result in additional expenses for the patient or his/her health coverage.

d) Lastly, the reasons given by health care providers in favor of interchangeability refer to costs. It is important to note that, in Argentina, according to the prices reported by Kairos Argentina, some biosimilar drugs are offered at a higher price than the original products. Therefore, it is noticed that, year after year, cost bids are random; hence, this could only be applied to patients who start their GH therapy knowing that, in the future, they would have to continue with the same product until finishing their treatment. See the annexes in electronic format.

Guillermo Alonso, M.D. ${ }^{a}$, Viviana Balbi, M.D. ${ }^{b}$, Cristina Bazán de Casella, M.D. ', Alicia Belgorosky, M.D. ${ }^{d}$, Ignacio Bergadá, M.D. ${ }^{e}$, Oscar Brunetto, M.D.f, Hamilton Cassinelli, M.D. ${ }^{e}$, Marta Ciaccio, M.D. ${ }^{d}$, Ana Keselman, M.D. ${ }^{e}$, Mirta B. Miras, M.D.g, Analía Morin, M.D. ${ }^{b}$ and National Committee of Pediatric Endocrinology. 
a. Hospital Italiano, Buenos Aires.

b. Hospital Sor María Ludovica, La Plata, Buenos Aires.

c. Hospital del Niño Jesús, Tucumán.

d. Hospital de Pediatría Juan P. Garrahan, Buenos Aires.

e. Hospital de Niños Ricardo Gutiérrez, Buenos Aires.

f. Hospital de Pediatría Pedro de Elizalde, Buenos Aires.

g. Hospital de Niños de la Santísima Trinidad, Córdoba.

E-mail address: Guillermo Alonso, M.D.:

guillermo.alonso@hospitalitaliano.org.ar

http: / / dx.doi.org/10.5546/ aap.2019.eng.213

To cite: Alonso G, Balbi V, Bazán de Casella C, Belgorovsky A, et al. Statement of Argentine pediatric endocrinologists on growth hormone interchangeability. Arch Argent Pediatr 2019;117(4):213-215.

\section{REFERENCES}

1. Parlement Européen et du Conseil. Directive 2004/27/ CE. 31 mars 2004. Journal officiel de l'Union européenne. 2004;136-34-57. [Accessed on:January $11^{\text {th }}, 2019$ ]. Available at: https: / / eur-lex.europa.eu/LexUriServ/LexUriServ.do?u ri=OJ:L:2004:136:0034:0057:FR:PDF.

2. Parlement Européen et du Conseil. Directive 2001/83/EC. Amended by Directives 2003/63/EC and 2004/. 6 november 2001. Journal officiel de l'Unioneuropéenne. 2001;311:67-128. [Accessed on: January 11 $\left.{ }^{\text {th }}, 2019\right]$. Available at: https:/ / ec.europa.eu/health / sites / health / files / files / eudralex / vol-1/dir_2001_83_consol_2012/dir_2001_83_cons_2012_ en.pdf.

3. European Medicines Agency. Guideline on Similar Biological Medicinal Products. CHMP / 437/ 04.30 october 2005. [Accessed on:January $11^{\text {th }}, 2019$ ]. Availableat: https: / / www.ema.europa.eu/documents / scientific-guideline/ guideline-similar-biological-medicinal-products_en.pdf.

4. European Medicines Agency. Guideline on Similar Biological Medicinal Products Containing Biotechnology-
Derived Proteins as. Active Substance: Quality Issues. 22 February 2006. [Accessed on: January 11 ${ }^{\text {th }}, 2019$ ]. Available at: https:/ /www.ema.europa.eu/documents/ scientific-guideline/guideline-similar-biologicalmedicinal-products-containing-biotechnology-derivedproteins-active_en.pdf.

5. European Medicines Agency. Guideline on Similar Biological Medicinal Products Containing BiotechnologyDerived Proteins as Active Substance: Non-Clinical and Clinical Issues. 18 december 2014. [Accessed on: January 11 $\left.{ }^{\text {th }}, 2019\right]$. Available at: https://www.ema.europa. eu / documents / scientific-guideline / guideline-similarbiological-medicinal-products-containing-biotechnologyderived-proteins-active_en-2.pdf.

6. European Medicines Agency, Heads of Medicines Agencies. Guideline on good pharmacovigilance practices (GVP): Product-or Population-Specific Considerations II Biological medicinal products. 4 August 2016. [Accessed on: January $\left.11^{\text {th }}, 2019\right]$. Available at: https://www.ema.europa. eu/documents / scientific-guideline/ guideline-goodpharmacovigilance-practices-gvp-product-populationspecific-considerations-ii_en-0.pdf.

7. Ministerio de Salud. Resolución 326/2002. [Accessed on: February $\left.12^{\text {th }}, 2019\right]$. Available at: http: / / servicios.infoleg. gob.ar/infolegInternet/anexos / 70000-74999/74926 / norma.htm

8. A.N.M.A.T. Ministerio de Salud de la Nación. Ley 25649. [Accessed on: February 12 $\left.{ }^{\text {th }}, 2019\right]$. Available at: http:/ / www.anmat.gov.ar/fna/25649.asp

9. Swerdlow AJ, Cooke R, Beckers D, Borgström B, et al. Cancer risks in patients treated with growth hormone in childhood: the SAGhE European cohort study. J Clin Endocrinol Metab. 2017;102(5):1661-72.

10. Poidvin A, Touzé E, Ecosse E, Landier F, et al. Growth hormone treatment for childhood short stature and risk of stroke in early adulthood. Neurology. 2014; 83(9):780-6.

11. Allen DB. Growth hormone post-marketing surveillance: safety, sales, and the unfinished task ahead.JClin Endocrinol Metab. 2010;95(1):52-5. 


\section{ANEXO I \\ Generalidades de los medicamentos biológicos}

De acuerdo con las recomendaciones de la European Medicines Agency (EMA), http: / / www.ema.europa.eu/docs/en_GB/document library / Leaflet/2017/05/ WC500226648.pdf, los siguientes conceptos resumen sus características para el fin de este documento.

\section{Grado de variabilidad inherente al proceso}

Los medicamentos biológicos son fabricados por organismos vivos, que son naturalmente variables. Por lo tanto, la sustancia activa en la medicina biológica final puede tener un grado inherente de variabilidad menor ("microheterogeneidad"). Esta debe estar dentro del rango aceptable para garantizar la seguridad y la eficacia constantes. Sin embargo, este grado de variabilidad menor puede estar presente dentro o entre lotes de la misma medicina biológica. Por ello, deben aplicarse siempre controles estrictos para asegurar que, a pesar de esta variabilidad, haya consistencia de lote a lote y que las diferencias no afecten la seguridad o la eficacia.

Para un medicamento terminado, tanto el medicamento biosimilar como el de referencia deben tener la misma posología y vía de administración. Se pueden permitir algunas diferencias si no tienen ningún efecto sobre la seguridad y la eficacia, a saber, diferencias en la formulación del medicamento (por ejemplo, excipientes), presentación (por ejemplo, polvo para reconstituir versus solución lista para inyección) y dispositivo de administración (por ej., pen, jeringa, etc.).

Los estudios para demostrar la calidad farmacéutica deben proporcionar datos detallados sobre lo siguiente: a) caracterización estructural y otras propiedades fisicoquímicas; b) pureza (las huellas de residuos del proceso de fabricación deben controlarse y no deben exceder los niveles aceptables); c) actividad biológica; d) excipientes y materiales de partida; e) fortaleza y formulación; f) el control del proceso de fabricación (para garantizar que la sustancia activa y el producto terminado se ajustan a los rangos aceptados para las especificaciones técnicas); g) estabilidad de la sustancia activa y del producto acabado durante la vida útil en condiciones de almacenamiento definidas.

La comparación de lotes antes y después de un cambio de fabricación debería garantizar la consistencia, de manera que no haya modificaciones en la seguridad o eficacia.

\section{Seguridad}

En los últimos 10 años, el sistema de monitoreo de la UE para cuestiones de seguridad no ha identificado ninguna diferencia relevante en la naturaleza, en la gravedad o en la frecuencia de los efectos adversos entre los medicamentos biosimilares y sus medicamentos de referencia.

Los estudios posteriores a la comercialización permiten el monitoreo de los riesgos conocidos y también la detección de reacciones adversas raras que surgen solo cuando un gran número de pacientes han sido tratados durante un período prolongado. Por esta razón, en el momento de la aprobación, los reguladores pueden imponer a la empresa la obligación de llevar a cabo un estudio de seguridad posterior a la autorización.

\section{Inmunogenicidad}

En la guía para buenas pautas de fármacovigilancia de productos biológicos, la $E M A$ $\mathrm{y}$ Heads of Medicines Agencies (HMA) ${ }^{6}$ hacen referencia a que, en el caso de los fármacos biológicos, la inmunogenicidad es el punto más importante para tener en cuenta en la fármacovigilancia a corto y a largo plazo. Refieren que, en la mayoría de los casos, no hay problemas relevantes; sin embargo, en ocasiones, la inmunogenicidad podría dar lugar a reacciones serias. La fuente de inmunogenicidad podría estar relacionada con factores vinculados al producto, la célula en la que se produce el fármaco, cambios postransduccionales y en la estructura tridimensional durante el procesamiento; factores relacionados con el tratamiento, la dosis y la vía de administración, y factores relacionados con el paciente, el bagaje genético, las medicaciones concomitantes, la naturaleza de la enfermedad de base y el estado inmunológico. Las consecuencias clínicas de la inmunogenicidad podrían incluir la pérdida parcial o total de la eficacia del producto o la alteración de la farmacocinética por la generación de anticuerpos neutralizantes o de efectos inmunes, como anafilaxia, formación de inmunocomplejos e inducción de reacción cruzada con proteínas endógenas u otros autoanticuerpos. 
Mencionan que, si bien se debe realizar una evaluación de inmunogenicidad pre autorización del fármaco, por el hecho de que la muestra de pacientes, usualmente, es pequeña y los efectos graves son poco frecuentes, no se puede evaluar con certeza la posibilidad de efectos a largo plazo. Esto hace necesaria la fármaco-vigilancia a largo plazo poscomercialización de cada producto biológico, sea este de referencia o similar.

\section{Trazabilidad del producto}

En la misma guía, ${ }^{6}$ hacen referencia a que los productos biológicos, al igual que cualquier otro medicamento, pueden sufrir variaciones en el proceso de fabricación luego del período de autorización. Esto hace necesario asegurar una continua trazabilidad del producto y de cada lote. Es esencial que diferentes productos con el mismo principio activo se puedan diferenciar, a fin de que nuevos aspectos de seguridad emergentes sean rápidamente detectados y evaluados durante el ciclo de vida del producto y puedan ser referenciados al lugar y al paciente. 


\section{ANEXO II \\ Estudios de intercambiabilidad de biosimilares}

\begin{abstract}
Hlavaty T \& Letkovsky J. Biosimilars in the therapy of inflammatory bowel diseases. Eur J Gastroenterol Hepatol. 2014 Jun;26(6):581-7.

El principal beneficio de los biosimilares antifactor de necrosis tumoral- $\alpha$ (anti-tumor necrosis factor $\alpha$; anti-TNFa, por sus siglas en inglés) sería el costo reducido del tratamiento, que dará como resultado un mejor acceso a la terapia biológica. Sin embargo, aún existen preocupaciones sobre la eficacia y la seguridad de los biosimilares y la intercambiabilidad libre de biosimilares y productos biofarmacéuticos originales. Si bien impresiona que los péptidos biosimilares (epoetinas y los factores de crecimiento), los perfiles de seguridad y de eficacia son comparables, la potencial inmunogenicidad sigue siendo una preocupación.
\end{abstract}

Ebbers HC, Muenzberg M, Schellekens H. The safety of switching between therapeutic proteins. Expert Opin Biol Ther. 2012 Nov; 12(11):1473-85.

La EMA ha emitido una declaración de que el cambio de medicamento solo debería ocurrir siguiendo las instrucciones de un profesional de la salud calificado. Muchos países europeos tienen diferentes tipos de políticas para frenar la sustitución automática de biosimilares en el nivel de la farmacia. Estas legislaciones y pautas, principalmente, restringen la sustitución por biosimilares durante un período limitado después de su introducción y están destinadas a aumentar la trazabilidad de posibles eventos adversos a productos específicos. Estas políticas, a veces, se interpretan como signos de que el cambio a los biosimilares podría ser inseguro.

Para comprender la dinámica del cambio de medicamentos en tiempo real y la ocurrencia de eventos de seguridad debido a este, se requieren más estudios sobre la práctica clínica del cambio.

Refieren que, hasta el año 2012, no habían surgido significativas señales de seguridad como resultado de cambiar a productos biofarmacéuticos, incluidos los biosimilares. Sin embargo, para garantizar que los prescriptores estén seguros del perfil de riesgo de los biosimilares, tanto ahora como en el futuro, son necesarios métodos robustos para monitorear la seguridad de los biosimilares posterior a su autorización de comercialización.
(The Alliance for Safe Biologic Medicines (ASBM). Interchangeability and Physician Notification. http: / / safebiologics.org/resources/in-the-states / . Accessed: 30 Sept 2016).

La intercambiabilidad es intrínsecamente más difícil de establecer para los biosimilares que para las versiones genéricas de las drogas químicas porque los productos biosimilares nunca pueden ser copias exactas de productos biológicos innovadores.

Kurki P, Van Aerts L, Wolff-Holz E, Giezen T, Skibeli V, Weise M. Interchangeability of Biosimilars: A European Perspective. Bio Drugs. 2017 Apr;31(2):83-91

En los últimos 10 años, la experiencia con biosimilares ha demostrado que incluso complejos, como las proteínas derivadas de la biotecnología, se pueden copiar con éxito. Esto ha desencadenado una discusión intensiva sobre la intercambiabilidad de un biosimilar con su producto de referencia, con la principal preocupación en la inmunogenicidad. Concluyen que cambiar entre versiones comparables de la misma sustancia activa aprobada de acuerdo con la legislación de la UE no se espera que desencadene o aumente la inmunogenicidad. De acuerdo con el conocimiento actual, es poco probable y muy difícil para corroborar que dos productos comparables tengan diferente seguridad o eficacia en pacientes individuales cuando se produce un cambio en la medicación.

Romer T, Zabransky M, Walczak M, Szalecki M, Balser S. Effect of Switching Recombinant Human Growth Hormone: Comparative Analysis of Phase 3 Clinical Data. Biol Ther. 2011;1(1):005.

El cambio de Genotropin a Omnitrope líquida no tiene un impacto negativo en la eficacia, en la seguridad y en la inmunogenicidad. No hubo diferencias significativas en la evolución del crecimiento ni en las concentraciones de factor de crecimiento similar a la insulina tipo 1 (insulin-like growth factor-1; IGF-1, por sus siglas en inglés) y la proteína transportadora 3 del factor de crecimiento similar a la insulina (insulin-like growth factor-binding protein-3; IGFBP-3, por sus siglas en inglés) en los niños tratados en forma continua con Omnitrope. El liofilizado y las 
preparaciones líquidas de Omnitrope fueron bien toleradas, y el cambio de medicamento no afectó en forma adversa la seguridad. Por lo tanto, las preparaciones Omnitrope y Genotropin son comparables. El cambio parece ser seguro (55 pacientes y 18 meses de seguimiento). El presente estudio incluye personal de Sandoz.

Rashid N, Saenger $P$, Wu YL, Woehling $H$, Frankel M, Lifshitz F, Muenzberg $M$, Rapaport R. Switching to Omnitrope ${ }^{\circledR}$ from Other Recombinant Human Growth Hormone Therapies: A Retrospective Study in an Integrated Healthcare System. Biologics in Therapy. 2014;4(1-2):27-39.

Este estudio utilizó datos retrospectivos del mundo real para examinar el impacto del cambio de una hormona de crecimiento recombinante humana (recombinant human growth hormone; $r h G H$, por sus siglas en inglés) no Omnitrope a Omnitrope. El estudio demostró que los pacientes continuaron creciendo sin alteración en sus trayectorias de crecimiento y, por lo tanto, pudieron pasar de una $r h G H$ que no fuera de Omnitrope a Omnitrope sin ningún impacto negativo en su crecimiento. Nuestros hallazgos deberían ser un recurso útil para los médicos que enfrentaran la posibilidad de cambiar de terapia con $r h G H$. El presente trabajo no emite ningún comentario sobre seguridad.

Flodmark CE, Lilja $K$, Woehling $H$, Ja"rvholm K. Switching From Originator to biosimilar Human Growth Hormone Using Dialogue Teamwork: Single-Center Experience From Sweden. Biol Ther. 2013;3:35-43.

La disponibilidad de medicamentos biosimilares tiene beneficios que pueden ser importantes para el cuidado de la salud de proveedores y pacientes en términos de reducción de los costos de las drogas y, posiblemente, del incremento del acceso de pacientes a los tratamientos.

En junio de 2009, por motivos económicos, se implementó un nuevo plan de tratamiento para los niños bajo tratamiento con $r h G H$. Esto implicó cambiar a los pacientes el tratamiento existente con $r h G H$ original al biosimilar (Omnitrope), utilizando un enfoque de trabajo de equipo de diálogo cuyo componente principal era la educación interprofesional. La viabilidad de usar este enfoque para implementar el cambio a un biosimilar fue evaluada, así como el impacto del cambio en la eficacia del tratamiento y el costo de la terapia. Duración del cambio: 12-18 meses.

Para implementar la estrategia, se ofreció a los pacientes y a sus padres lo siguiente:

- Una carta del jefe del Departamento de Pediatría en la que se explicaba la razón económica para el cambio.

- La oportunidad de discutir sobre aspectos del cambio con el médico responsable del cuidado del paciente.

- En caso de la no aceptación, se ofrecía un diálogo adicional con el jefe del Departamento.

- Información sobre la rhGH biosimilar (somatrofina) por las autoridades regulatorias.

- Una visita a una enfermera especializada en Endocrinología para recibir instrucciones sobre cómo actuaba el aplicador (por ejemplo, usar el nuevo dispositivo) y para hacer cualquier pregunta sobre el cambio.

- El número de teléfono del especialista o enfermera de Endocrinología para contactarse en busca de consejo.

Conclusiones. El trabajo de equipo en diálogos se puede utilizar para lograr cambiar pacientes de una $r h G H$ original a biosimilares, sin efectos negativos en el crecimiento de los pacientes ni eventos adversos (EA) graves inesperados. Esta estrategia está asociada con ahorros de costos significativos.

Comentario. Los autores refieren que, sobre la base de este estudio, se formula la hipótesis de que hay cuatro elementos críticos para el éxito de ese enfoque, que lleva a un cambio en las interacciones entre los profesionales de la salud: (1) proporcionar a los pacientes información clara sobre los motivos del cambio; (2) permitir a los pacientes / cuidadores individualmente suficientes oportunidades para discutir el cambio con los diferentes profesionales de la salud involucrados; (3) un enfoque de equipo conjunto que evite mensajes mixtos de los diferentes servicios de salud profesionales involucrados; (4) proporcionar pacientes con la tranquilidad del apoyo personal a lo largo del cambio.

"Los autores refieren que cambiar por un diferente producto de $r h G H$ también implica el uso de un nuevo dispositivo de inyección. En consecuencia, los pacientes deben aprenderlo y acostumbrarse a una inyección de diferente técnica"'.

El presente trabajo está subsidiado por Sandoz. 
Editorial sobre el trabajo de Flodmark y col. Mats Ekelund - Christopher Bidad - Roy Gomez. To the Editor; A Commentary on "Switching From Originator to Biosimilar Human Growth Hormone Using a Dialogue Teamwork: Single-Center Experience From Sweden". Biol Ther.2014;4:69-71.
El trabajo tiene muchas limitaciones metodológicas y de interpretación que, junto con la falta de una nota de precaución sobre el peligro de la sobreinterpretación del resultado de este estudio no intervencionista, plantea preguntas sobre la validez del estudio y sus conclusiones.

(Advertencias: dos de los tres autores de esta editorial son empleados de Pfizer). 